\title{
Corela
}

Cognition, représentation, langage

12-1 | 2014

Vol. $12, \mathrm{n}^{\circ} 1$

\section{Enough et too : expression de la suffisance et de l'excès dans les constructions «tough » en anglais}

\author{
Joasha Boutault
}

\section{CpenEdition}

\section{Journals}

Édition électronique

URL : http://journals.openedition.org/corela/3461

DOI : $10.4000 /$ corela.3461

ISSN : 1638-573X

Éditeur

Cercle linguistique du Centre et de I'Ouest - CerLICO

Référence électronique

Joasha Boutault, «Enough et too : expression de la suffisance et de l'excès dans les constructions « tough » en anglais », Corela [En ligne], 12-1 | 2014, mis en ligne le 25 juin 2014, consulté le 30 avril 2019. URL : http://journals.openedition.org/corela/3461 ; DOI : 10.4000/corela.3461

Ce document a été généré automatiquement le 30 avril 2019

\section{(c) (i) (2)(2)}

Corela - cognition, représentation, langage est mis à disposition selon les termes de la licence Creative Commons Attribution - Pas d'Utilisation Commerciale - Partage dans les Mêmes Conditions 4.0 International. 


\title{
Enough et too: expression de la suffisance et de l'excès dans les constructions « tough » en anglais
}

\author{
Joasha Boutault
}

\section{NOTE DE L'AUTEUR}

Je remercie les deux relecteurs anonymes, dont les remarques ont permis d'enrichir la version initiale de cet article.

\section{Introduction}

1 Les constructions « tough $»^{1}$, telles que this manuscript is easy to read, constituent l'une des stratégies à la disposition des langues ${ }^{2}$ pour mettre en relief et prédiquer une propriété d'un objet dans une structure phrastique. Ces constructions sont composées de manière prototypique d'un sujet nominal, d'un verbe copule qui prend pour complément un groupe adjectival formé d'un adjectif et d'un verbe à l'infinitif. Le sujet grammatical, marqué au nominatif ${ }^{3}$, est interprété comme co-référent de l'argument interne du verbe infinitif. Pour cette raison, les constructions «tough" sont souvent analysées ${ }^{4}$ en grammaire générative comme dérivées par montée de l'argument interne vers la position de spécifieur de IP, c'est-à-dire de sujet grammatical :

(1) John $n_{i}$ is easy to please $t_{i}$.

2 Le problème est ici que John reçoit deux cas : l'accusatif lorsqu'il est en position d'objet de please, et le nominatif lorsqu'il remonte en position de sujet. Ceci est problématique dans le cadre de la grammaire générative, puisque le filtre sur les cas interdit à tout argument d'avoir plus d'un cas 5 .

3 Les constructions « tough " sont à différencier des structures ci-dessous :

(2) Susan is likely to believe your story. 
(3) Paul is ready to leave.

4 Tandis que (1) constitue une structure à montée "object-to-subject ", (2) présente une montée du sujet du verbe infinitif vers la position de sujet du prédicat adjectival be likely (subject-to-subject raising) :

(4) Susan ${ }_{i}$ is likely to $t_{i}$ believe your story.

Be likely a pour seul argument la proposition infinitive, et Susan est argument externe de believe.

6 (3), en revanche, est une structure dite à contrôle du sujet, dérivée comme suit:

(5) Paul is ready to $\mathrm{PRO}_{\mathrm{i}}$ leave.

7 Ici, Paul est argument à la fois du prédicat adjectival be ready et du verbe leave et reçoit par conséquent un rôle de chacun d'entre eux. Or, en grammaire générative, ceci viole le Critère Thématique ${ }^{6}$, qui stipule que tout argument ne peut recevoir qu'un seul rôle. PRO, pronom phonétiquement nul et non marqué casuellement, est inséré dans la structure pour porter le rôle excédentaire, attribué par le verbe infinitif.

Par essence, les constructions «tough » dénotent une évaluation de la part du locuteur. Pour cette raison, elles doivent obligatoirement contenir un élément qui apporte cette évaluation. Dans la majorité des cas, c'est un adjectif évaluatif qui joue ce rôle :

(6) The decision was easy to make. ${ }^{7}$

(7) Kenneth Branagh is great to work with. ${ }^{8}$

9 La notion d'adjectif évaluatif utilisée ici correspond aux adjectifs modaux de Rivière (1983), dont il donne la définition suivante :

The adjectives under study will be defined as modal : they denote an attitude of the speaker toward the proposition he utters.

Rivière $(1983: 2)$

10 L'évaluation exprimée par les adjectifs dits « évaluatifs » correspond donc à l'expression d'un point de vue subjectif concernant une propriété attribuée à un argument.

11 A ceux-ci s'opposent les adjectifs descriptifs ${ }^{9}$, dont l'interprétation se veut plus objective puisqu'ils décrivent des propriétés constatables. Leur utilisation dans les constructions « tough » est sujette à la présence d'une modification supplémentaire de type adverbial :

(8) Brutha tugged at the piece of curved pottery. It had been too broken for anyone to loot. ${ }^{10}$

(9) * It had been broken for anyone to loot.

(10) Most of the hides were well cured and stretched, so that it was a matter of finding one the proper size and thinness for her drum frame. She softened the chosen hide in the tub of water, working the skin in her hands until it was flexible enough to draw across the frame. ${ }^{11}$

(11) *... until it was flexible to draw across the frame.

12 L'étude ${ }^{12}$ d'un corpus de 1050 énoncés «tough » authentiques a montré que seuls too et enough permettent d'utiliser un adjectif descriptif dans une telle structure :

(12) * It had been very broken for anyone to loot.

(13) *... until it was very flexible to draw across the frame.

13 La question est de savoir ce qui, dans ces deux adverbes, rend licite un adjectif descriptif. Qu'apportent-ils du point de vue sémantique? En quoi permettent-ils d'exprimer l'évaluation indispensable aux constructions «tough »? Pour répondre à ces questions, nous étudierons des énoncés «tough » de plusieurs types : avec adjectif évaluatif comme avec adjectif descriptif, ainsi que présentant différents comportements syntaxiques. 


\section{Enough: accès au validable}

appellent « compromisers $»^{16}$ et à laquelle appartient enough comme indiquant le fait que le procès décrit par le verbe n'est pas approprié. Dans les constructions « tough », c'est le degré exprimé par l'adjectif qui est considéré comme inapproprié. Ainsi, dans l'énoncé (14) ci-après, le référent du sujet possède la propriété exprimée par l'adjectif cool à un degré suffisant (symbolisé par le trait vertical représentant la frontière entre le domaine $\mathrm{du}$ validable et le domaine du non validable) pour permettre la validation de la relation prédicative. To construit une occurrence quelconque de DRINK qui est posée comme atteignable :

(14) The tea was cool enough to drink. ${ }^{17}$

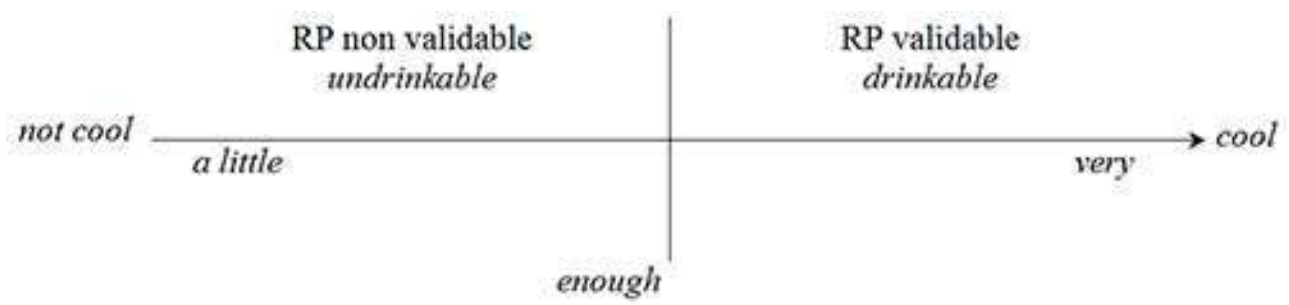

Enough représente un degré qui fonctionne comme une frontière entre deux domaines : le domaine à l'intérieur duquel la relation prédicative est validable (I) et celui où elle ne l'est pas $(E)^{18}$. Il s'agit d'un degré adéquat. Cet adverbe constitue donc un outil d'accès au domaine du validable ${ }^{19}$. Il en va de même dans les énoncés ci-dessous :

(15) Your mother isn't important enough to say hello to ?20

(16) We're looking for a thirty to forty year old male veteran driving a car large

enough to shoot from but not so large it was noticed..$^{21}$ 
(17) Fast days and feast days and Prophets' Days and bread days... who cares ? A God whose only concern about food now is that it's low enough to reach ?22 donc indispensable pour préciser les circonstances dans lesquelles la propriété est attribuée. Cependant, au delà du fait que pretty et ugly sont des antonymes, il ne s'agit pas du même adjectif : la différence entre beauté physique et beauté morale est ici capitale. L'adverbe inside ne peut pas être supprimé parce que, comme la proposition infinitive, il spécifie les circonstances dans lesquelles l'adjectif qualifie le référent du sujet. En son absence, ugly serait interprété comme antonyme de pretty au sens de beauté physique, ce qui rendrait l'énoncé absurde :

(20) *Mary is sure pretty to look at, but she's ugly to look at.

En revanche, il est tout à fait possible d'effacer le complément infinitif de pretty: 
est possible car le référent de Mary ne peut être qualifié de pretty que parce qu'il est perçu comme tel. Dans des cas comme celui-ci, la proposition infinitive est pratiquement redondante et de ce fait, on peut s'en dispenser. Ceci évoque l'«objet interne » de Franckel et Paillard (1992:33):

Les exemples (1) [Il a écrit la lettre que Paul lui réclame depuis trois semaines] et (2) [Il a écrit des lettres] présentent en même temps une propriété commune: l'écriture y est spécifiée comme étant "l'écriture de quelque chose" (de type lettre ou roman). Cette prise en compte de "l'écriture de quelque chose" s'impose même en l'absence d'un $C_{1}$ explicite, comme dans :

(3) Il a écrit.

Cet exemple fait en effet apparaître que la localisation de écrire dans le temps entraîne par elle-même la présence d'un objet minimal, objet inscrit dans le prédicat lui-même, traditionnellement appelé objet interne, et formulable en termes d'écrivable.

Dans le cas des constructions «tough », il ne s'agit pas de la relation entre le prédicat et son complément, mais de l'adjectif et de la proposition infinitive qui le complète. La notion exprimée par le verbe look at est inscrite dans l'adjectif pretty. Si on veut faire référence à une autre notion que celle-ci, alors il faut le mentionner, ce qui est fait dans les constructions « tough » par le biais de l'infinitive.

Les énoncés ci-après ne se comportent pas du tout de la même façon que (14) à (17) : l'infinitive n'est pas effaçable (b) mais l'antéposition est possible (c), de même que la création d'une variante avec un pronom explétif pour sujet (d) :

(22) a. He told Egil to take him to the place where he'd last seen the two men. They were easy enough to track down. ${ }^{27}$

b. "They were easy enough.

c. To track them down was easy enough.

d. It was easy enough to track them down.

(23) a. Mr Cut-Me-Own-Throat Dibbler, Purveyor, Merchant Venturer and all-round salesman, had thought long and hard about going into ethnic foodstuff. But it was a natural career progression. The old sausage-in-a-bun trade had been falling off lately, while there were all these trolls and dwarfs around with money in their pockets (...). Dwarfs were easy enough to cater for. Rat-on-a-stick was simple enough, although it meant a general improvement in Dibbler's normal catering standards. $^{28}$

b. "Dwarfs were easy enough.

c. To cater for dwarfs was easy enough.

c. It was easy enough to cater for dwarfs.

(24) a. The idea of her father or Jeff getting inside her head in so intimate a manner was difficult enough to contemplate. The idea of Mik being there as well was different. ${ }^{29}$

b. "The idea (...) was difficult enough.

c. To contemplate this idea (...) was difficult enough.

d. It was difficult enough to contemplate this idea.

(25) a. 'I'll eat as fast as I can, Tom, and get back on the board.'

'Take your time,' Rawlins did not take his eyes off the monitor. 'No sense getting ulcers ; you're hard enough to live with, as it is.' ${ }^{30}$

b. "You're hard enough.

c. To live with you is hard enough.

d. It is hard enough to live with you.

Les adjectifs de ces exemples ne modifient le sujet grammatical que dans son implication dans l'évènement exprimé par le procès. Si l'adjectif ne modifie pas directement le sujet, il est impossible de dire que le référent de ce dernier possède la propriété exprimée par 
l'adjectif à un degré adéquat permettant la validation de la relation prédicative. Ainsi, enough modifie l'ensemble adjectif + proposition infinitive, c'est-à-dire la propriété attribuée à un argument dans son implication dans le procès décrit par le verbe infinitif. Ceci est tout particulièrement visible dans les énoncés (e) ci-dessous :

(22) a. They were easy enough to track down.

e. "They were sufficiently easy to track down.

(23) a. Dwarfs were easy enough to cater for.

e. "It was sufficiently easy to cater for dwarfs.

(24) a. The idea of her father or Jeff getting inside her head in so intimate a manner

was difficult enough to contemplate.

e. "It was sufficiently difficult to contemplate this idea.

(25) a. You're hard enough to live with, as it is

e. "It was sufficiently hard to live with you.

La substitution de enough par sufficiently ne permet pas de conserver le sens. Dans de tels exemples, l'infinitive ne peut donc pas constituer le repère-comparant puisque celui-ci doit rester extérieur à la modification. ${ }^{\text {Il }}$ reste par conséquent implicite. On pourra noter que tous les adjectifs des exemples (22) à (25) sont évaluatifs. Les études de corpus montrent en effet que seuls de tels adjectifs adoptent ce comportement dans les constructions " tough " $^{31}$. Il est ainsi possible de faire le lien avec l'emploi « absolu » des adjectifs, dont R. Rivara (1993:41), cité par C. Moreau (2006 :note 2), donne la définition suivante :

Les emplois dits (syntaxiquement) absolus des adjectifs (grand, mais aussi très grand) sont caractérisés par l'absence de référence à un comparant. Ils semblent véhiculer une information " absolue » (non relative à un repère). (...) Il est d'autre part inhérent au fonctionnement absolu des adjectifs graduables que les normes qu'ils mettent en jeu sont implicites. (...)

Or, les jugements renfermant des adjectifs graduables employés absolument sont toujours exposés à la contestation, au sens où le sont, mutatis mutandis, les jugements de valeur, et ils sont, par là, de type évaluatif.

On peut ainsi comparer ces exemples à (26) ci-dessous, emprunté à C. Moreau (2006 :7) :

(26) She seems happy enough.

Elle propose de gloser cet énoncé par « elle semble heureuse finalement, assez pour dire qu'elle n'est pas malheureuse ». On effectue ici une opération de centrage notionnel : en l'absence de repère-comparant, la comparaison se fait par rapport au centre organisateur 32. L'utilisation de l'adverbe enough a pour effet d'atténuer la propriété exprimée par l'adjectif. De cette manière, on exprime le fait que le nombre de propriétés correspondant à la notion (ici HAPPY) est suffisamment important pour que l'on puisse considérer que l'énoncé exprime une occurrence de cette notion.

31 C'est également ce qui se passe dans les constructions «tough" (22) à (24) reprises cidessous, et qui, rappelons-le, contiennent un adjectif évaluatif. L'adverbe enough exprime le fait que les propriétés dénotées par le groupe adjectif + proposition infinitive ne sont pas suffisamment nombreuses pour que référence soit faite au plus haut degré de la notion correspondante :

(22) They were easy enough to track down.

(23) Dwarfs were easy enough to cater for.

(24) The idea of her father or Jeff getting inside her head in so intimate a manner was difficult enough to contemplate.

(25) You're hard enough to live with. 
Ainsi, en (22), les hommes en question sont effectivement faciles à pister, mais pas autant qu'ils pourraient l'être. Ce qui est pertinent dans ce type d'exemple n'est pas la validabilité de la relation prédicative mais plutôt la définition de la notion exprimée par l'ensemble adjectif + proposition infinitive.

\section{Too : outil de blocage}

Si enough exprime la suffisance, too, pour sa part, exprime un degré situé au-dessus d'une norme et traduit l'excès. En (27) ci-dessous ${ }^{33}$, le personnage Poldarn est atteint d'amnésie. La construction « tough » exprime l'impossibilité d'effectuer l'action explain :

(27) 'Would you mind telling me who you are, by the way? I'm sure I should know you, but I've forgotten.' (...)

'More teasing. If you don't stop, I'm leaving.'

'No really', Poldarn sighed. 'It's too complicated to explain, but I promise you, I really don't remember.' ${ }^{34}$

On peut représenter l'échelle de complexité mise en jeu de la manière suivante :

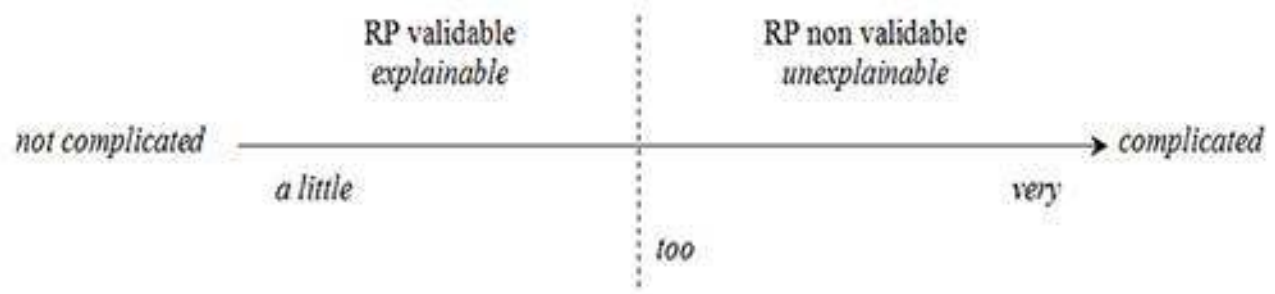

Cette échelle part de «not complicated » et se prolonge jusqu'à « complicated ». Plus on déplace le curseur de complexité vers la frontière, moins la relation prédicative est susceptible d'être validée. En (27), la frontière représentant le degré adéquat est dépassée. Too exprime donc le fait que le référent du sujet possède la propriété « complicated » à un degré tel qu'il bloque la validation de la relation prédicative. Il est intéressant de comparer le schéma ci-dessus à celui utilisé en (14) pour représenter l'énoncé The tea was cool enough to drink. On déplaçait alors le curseur depuis $\mathrm{E}$ jusqu'à I, s'approchant de la frontière représentée par enough et permettant l'accès au domaine du validable, à l'inverse de too qui lui, marque l'entrée dans le domaine du non validable. Too constitue un outil de blocage ${ }^{35}$ que la négation de cet adverbe lève, permettant ainsi l'accès au validable :

(28) It is not too complicated to explain.

(29) The tea is not too hot to drink.

Il existe des énoncés dans lesquels la présence de la négation a un effet différent :

(30) 'I've been looking for you.'

'And you found me. You must tell me how you do it. I haven't found anything I've

been looking for since I set foot in this bloody town.'

'You weren't too hard to find. (... $)^{36}$

(31) That said, he could close his eyes and it'd be the same river. That wasn't too

hard to arrive at, if he supposed that the Bohec ran into the sea (...). ${ }^{37}$

Too n'a pas ici une valeur d'excès et d'outil de blocage mais plutôt celle de l'intensifieur very.

Les adverbes almost et nearly (et near) semblent, à première vue, se comporter de manière inverse à too : la frontière dont le franchissement permettrait la validation de la relation 
prédicative n'est pas atteinte. L'étude des exemples montre que le fonctionnement de ces deux adverbes n'est pas exactement le même que celui de too :

(32) I discovered this fascinating old manuscript, so faded and worn it was almost impossible to read. ${ }^{38}$

(33) 'With psychotic killers... They're guided by a given delusion.'

'ok, so until we understand the nature of the delusion, we can't predict his next move.'

'And that's nearly impossible to do. ${ }^{39}$

(34) Janco threw one of the reservists into the group of spectators. A wild delight shone on his face as cheers and jeers rose to deafening levels. Ari rubbed his hand over his eyes. His partner would be near impossible to be with after tonight. To keep his gloating to a minimum, Ari hoped someone would give Janco a close match. ${ }^{40}$

(35) As soon as his eyelids closed, he knew he was somewhere else, in a dream; he was sure of that, because he was sitting at a table, in the middle of which stood a wonderfully lifelike ebony statue of a crow with a ring in its beak. It was extraordinarily realistic; in fact, it looked more like a crow than any crow he'd ever seen, especially the live ones. The urge to throw a doughnut at it was almost impossible to resist. ${ }^{41}$

(36) At this time the termites congregate in their eastern galleries. As the sun rises and the day warms up, the outer surface of the hill may become so hot that it is almost painful to touch, but the full strength of the midday sun is minimised since all it strikes directly is the thin top edge of the hill. ${ }^{42}$

Ces énoncés expriment le fait que la frontière n'est pas atteinte, c'est-à-dire que l'on se trouve encore à l'intérieur du domaine du possible. On peut noter qu'un autre paramètre entre en jeu dans les énoncés (32) à (36), qui contiennent l'adjectif impossible.

En (27) It's too complicated to explain but (...) I really don't remember que nous avons commenté plus haut, la validabilité de la relation prédicative est dépendante du degré associé à la propriété dénotée par l'adjectif. Dans les exemples contenant impossible ${ }^{43}$, c'est de l'adjectif lui-même que dépend la validabilité de la relation. Il n'est pas question ici d'un gradient de possibilité, il ne s'agit pas de dire si le degré de possibilité adéquat est atteint ou non. La propriété dont dépend la validation potentielle de la relation est exprimée non pas par la construction « tough » mais par le contexte. Dans l'exemple (32), les adjectifs faded and worn sont modifiés par l'adverbe so. Ce dernier agit de la même manière que too: il indique l'excès, le fait que la propriété décrite par l'adjectif a atteint un degré tel qu'elle bloque la validation de la relation prédicative exprimée par la construction « tough » complément de l'adjectif.

Dans les exemples (33) That's nearly impossible to do et (34) His partner would be near impossible to be with, la propriété pertinente n'est pas directement exprimée. Il est cependant possible de la déduire du contexte :

(33) b. And understanding the nature of the delusion is so difficult that [it]'s nearly impossible to do.

(34) b. His partner would be so smug that he would be near impossible to be with.

La situation est la même avec almost en (35) The urge to throw a doughnut at it [the statue of a crow] was almost impossible to resist. Ici, le personnage principal ne supporte pas les corbeaux. La raison pour laquelle le désir en question est presque irrépressible est liée à une propriété de la statue de l'oiseau: elle est si réaliste qu'elle donne envie au personnage de la chasser, comme il le ferait avec un véritable corbeau. On pourrait donc utiliser la glose suivante : 
(35) b. The statue was so lifelike that the urge to throw a doughnut at it was almost impossible to resist. comme sujet grammatical. C'est pour cette raison que les adjectifs utilisés sont généralement évaluatifs. Il est cependant possible de trouver des adjectifs purement descriptifs, tels que broken ou flexible, auquel cas, ils sont obligatoirement modifiés par un adverbe. C'est alors ce dernier qui apporte l'évaluation requise et obligatoirement exprimée par la structure. Cependant, seuls too et enough permettent de rendre licites les adjectifs descriptifs. Lorsque ces adverbes sont présents, la propriété exprimée par l'adjectif est évaluée par rapport à une norme, un étalon généralement représenté par la proposition infinitive. Cette dernière sert donc de repère-comparant à partir duquel la relation verbale est évaluée. Le degré exprimé par l'adverbe est alors jugé adéquat ou non. Avec too, outil de blocage, la propriété est évaluée comme excédant la norme, rendant impossible la validation de la relation prédicative. Si too est associé à une négation, la propriété est en-deçà de la norme et la relation validable. Enough, au contraire, indique que le degré souhaité est atteint, ce qui permet la validation (ou bien l'empêche si l'adverbe est accompagné d'une négation). Il constitue alors un outil d'accès au domaine du possible.

\section{BIBLIOGRAPHIE}

Boutault, J. (2012). A Hard Nut to Crack - Mouvements syntaxiques et motivation sémantique en anglais contemporain : les constructions « tough » et moyennes. Thèse de doctorat, Université de Poitiers. 
Charreyre, C. (1997). « La gradabilité est-elle donnée ou construite ? ». In C. Rivière and M.-L. Groussier (eds.), La notion. Gap, Ophrys. p. 53-59.

Cinque, G. (1999). Adverbs and Functional Heads : a Cross-Linguistic Perspective, Oxford Studies in Comparative Syntax. Oxford, Oxford University Press.

Culioli, A. (1985). Notes du séminaire de D.E.A : 1983-1984. Université de Poitiers.

Culioli, A. (1990). Pour une linguistique de l'énonciation : Opérations et représentations (T1). Paris, Gap, Ophrys.

Fleisher, N. (2006). "Attribution and Evaluation : The Syntax and Semantics of Attributive-withInfinitive Constructions". Berkeley, University of California.

Franckel, J., Paillard, D. (1992). « Objet : construction et spécification d'occurrences ». Le gré des langues, vol. 4. Paris, L'Harmattan. pp. 29-43.

Haegeman, L. (1991). Introduction to Government and Binding Theory. Oxford, Blackwell.

Mellet, S. (2009). « La frontière notionnelle en langue et en discours ». Cahiers de praxématique [En ligne], $\mathrm{n}^{\circ}$ 53. URL : http://praxematique.revues.org/1083.

Moreau, C. (2005). « Enough le degré qui rend possible », Intensité, Comparaison, Degré. Travaux du Cerlico, vol. 2.

Moreau, C. (2006). «Altérité et valuation dans les emplois absolus de enough et too ». Cycnos, vol. $23, n^{\circ} 1$.

Postal, P. (1971). Cross-over Phenomena. New York, Holt, Rinehart and Winston (Transatlantic Series in Linguistics).

Quirk, R., Greenbaum, S., Leech, G., and Svartvik, J. (1985). A Comprehensive Grammar of the English Language. London, Longman.

Rivara, R. (1993), « Adjectifs et structures sémantiques scalaires ». L'information grammaticale, vol. $58, \mathrm{n}^{\circ}$ 1. pp. 40-46.

Rivière, C. (1983). "Modal Adjectives : Transformations, Synonymy, and Complementation". Lingua, vol. 59. pp. 1-45.

Rosenbaum, P. (1967). The Grammar of English Predicate Complement Constructions, MIT Research Monograph Series. MIT Press.

\section{NOTES}

1. Cet article ne s'inscrit pas dans un cadre théorique unique. Il fait appel à des concepts divers issus de théories linguistiques différentes (en particulier la grammaire générative et la Théorie des Opérations Enonciatives) de façon à utiliser les outils les plus appropriés à la description des phénomènes étudiés.

2. On trouve des structures identiques ou proches des constructions «tough » dans des langues aussi variées que le français, l'allemand, le japonais, le coréen ou encore l'hébreu.

3. La morphologie casuelle en anglais est très limitée mais elle est bien visible sur les pronoms : I, me, my, mine, etc.

4. P. Rosenbaum (1967), P. Postal (1971) par exemple.

5. Voir, par exemple, L. Haegeman (1991:167).

6. Voir, par exemple, L. Haegeman (1991:54).

7. Michael Moorcock, Elric of Melniboné, ACE Charter, 1987, p. 28. 
8. http://forums.superherohype.com/showthread.php?p=17743125

9. Deux tests ont été appliqués aux adjectifs du corpus de façon à déterminer s'ils étaient descriptifs ou évaluatifs :

GN find GN adj 鉋 I find this book fascinating *I find this man disfigured

Arguably adj 廨 This book, arguably fascinating, is... *This man, arguably disfigured...

Ces résultats prouvent que fascinating est évaluatif et que disfigured est descriptif. (J. Boutault (2012)).

10. Terry Pratchett, Small Gods, Corgi, 1993, p. 279.

11. Anne McCaffrey, Dragonsinger : Harper of Pern, Corgi, 1978, p. 114.

12. J. Boutault (2012).

13. C. Moreau (2005:195).

14. Il s'agit d'une étude sur ce que Fleisher nomme "attributive-with-infinitive constructions ». Ces structures correspondent aux constructions «tough » du type de The Long Gallery would be a bugger to carpet (Terry Pratchett, Lords and Ladies, Corgi, 2001, p. 120), qui ont été écartées de la présente étude.

15. Fleisher explique que long dans "Middlemarch is a long book to read in one sitting " est intersectif parce qu'il modifie directement le nom tête (book), contrairement aux adjectifs tels que easy qui portent sur la relation exprimée par la subordonnée infinitive.

16. Les "compromiseurs" constituent une sous-catégorie des «downtoners", nos adverbes atténuatifs.

17. Karen Miller, Empress, Orbit, 2007, p. 26.

18. C. Moreau (2005:197).

19. C. Moreau $(2006: 14)$.

20. Maria V. Snyder, Storm Glass, Mira Novel, 2009, p. 100.

21. Criminal Minds, 'L.D.S.K', 106, Ernest Dickerson, 2006.

22. Terry Pratchett, Small Gods, Corgi, 1993, p. 148.

23. K.J. Parker, Memory, Orbit, 2003, p. 349.

24. Le contexte est ici très important. Le personnage principal, Opal, est souffleur de verre mais elle n'a pas pu exercer ses talents depuis longtemps. De retour chez ses parents après une longue absence, la première chose qu'elle fait est d'aller travailler dans l'atelier familial, sans même prendre le temps de saluer sa mère. Une paraphrase possible serait: "you don't think your mother is very important so you wait a very long time before coming to greet her ».

25. Nous considérons que de tels énoncés constituent bien des constructions « tough » puisqu'au delà de leur structure interne, ils présentent les mêmes particularités en terme d'attribution des cas.

26. Voir J. Boutault (2012:27-43) pour une discussion poussée sur les différentes possibilités en termes de comportement syntaxique des adjectifs des constructions « tough ».

27. K.J. Parker, Pattern, Orbit, 2003, p. 563.

28. Terry Pratchett, Men at Arms, Corgi, 2003, p. 189.

29. Marion Zimmer Bradley, Exile’s Song, Science Fiction Daw, 1996, p. 355.

30. Marion Zimmer Bradley, The Best of Marion Zimmer Bradley, 'Hero's Moon', edited by Martin H. Greenberg, A Sphere Book, 1990, p. 277.

31. En revanche, les adjectifs qui se comportent comme cool en (14) peuvent aussi bien être évaluatifs que descriptifs. Il n'existe donc pas de correspondance stricte du type d'adjectif (évaluatif ou descriptif) avec le comportement syntaxique vis-à-vis de l'infinitive des constructions « tough».

32. «Cela veut dire que lorsque vous avez des occurrences phénoménales, vous les ramenez à un type qui est le prédicat par excellence qui représente presque l'archétype platonicien. » A. Culioli (1985:27). 
33. Cet énoncé a un fonctionnement syntaxique différent des exemples (14) à (17) et (22) à (25) puisqu'ils acceptent toutes les manipulations :

(27) b. It is too complicated.

c. To explain it is too complicated.

d. It is too complicated to explain it.

Voir J. Boutault (2012).

34. K. J. Parker, Pattern, Orbit, 2003, p. 229.

35. C. Moreau (2006:10).

36. Steven Brust, Jhegaala, Tor Books, 2008, p. 128.

37. K.J. Parker, Pattern, Orbit, 2003, p. 16.

38. Marion Zimmer Bradley, The Shadow Matrix, DAW Books, 1997, p. 183.

39. Criminals Minds, 'Blood Hungry', 111, Charles Haid, 2005.

40. Maria V. Snyder, Power Study, http://www.mariavsnyder.com/powerstudy.php

41. K.J. Parker, Pattern, Orbit, 2003, p. 517.

42. BNC F9F.

43. Ces énoncés ont un fonctionnement syntaxique similaire à celui des énoncés (22) à (25) :

b. *[This fascinating old manuscript] was almost impossible.

c. To read [this fascinating old manuscript] was almost impossible.

d. It was almost impossible to read [this fascinating old manuscript].

44. S. Mellet (2009).

\section{RÉSUMÉS}

Les constructions «tough" (The decision was easy to make) sont des structures qui permettent d'exprimer une propriété du sujet, interprété comme co-référent de l'argument interne du verbe infinitif. Elles traduisent le point de vue du locuteur et à ce titre doivent obligatoirement contenir une forme d'évaluation. C'est l'adjectif qui joue généralement ce rôle, notamment dans des énoncés classiques tels que they will be difficult to convince dans lequel difficult est lui-même évaluatif. Lorsque l'adjectif est descriptif, comme c'est le cas dans this area is too spread out to defend, la présence de too ou de enough est indispensable. C'est alors cet adverbe qui apporte l'évaluation nécessaire à l'interprétation. A travers l'étude d'exemples authentiques, cet article montre que lorsqu'ils sont utilisés dans des constructions "tough", too et enough constituent respectivement des outils de blocage et d'accès au domaine du validable. Le point de vue du locuteur en ce qui concerne la validabilité de la relation prédicative est ainsi exprimé.

In English, the structures known as "tough" constructions (The decision was easy to make), enable the speaker to predicate a property of the subject, interpreted as co-referent of the internal argument of the infinitival verb. Therefore, some kind of evaluation has to be present. In "classical" examples such as they will be difficult to convince, the adjectives play this role : difficult is evaluative in itself. When the adjective is descriptive, as in this area is too spread out to defend, the presence of too or enough is compulsory. In those cases, the adverb is what provides the necessary evaluation. Through the study of authentic examples, this article shows how too and enough contribute to the evaluative interpretation of "tough" constructions. When they are used in such structures, these adverbs respectively prevent and enable the realisation of the event denoted by 
the infinitival verb. The point of view of the speaker on the possibility for the predicative relation to be validated is thus expressed.

INDEX

Mots-clés : évaluation, modalité, modification, validation, frontière

Keywords : evaluation, modality, modification, validation, boundary

\section{AUTEUR}

JOASHA BOUTAULT

FoReLL A - EA3816, Université de Poitiers 